\title{
Scalable Energy Management for Low Voltage Microgrids Using Multi-Agent Storage System Aggregation
}

\author{
Thomas Morstyn, Member, IEEE, Andrey V. Savkin, Senior Member, IEEE, \\ Branislav Hredzak, Senior Member, IEEE and Hoang D. Tuan, Member, IEEE
}

\begin{abstract}
This paper proposes multi-agent energy storage system aggregation as a means of scaling energy management to low voltage microgrids with distributed energy storage systems. Based on this concept, a hierarchical control strategy is developed for an AC microgrid with distributed battery and ultracapacitor energy storage systems. On the tertiary control level, the energy management problem is made scalable by considering each type of energy storage system in aggregate. This addresses the 'curse of dimensionality', since additional energy storage systems do not increase the optimisation problem dimension, and allows nonlinear energy storage models to be used for optimisation, accounting for variable efficiency, self-discharge and lifetime degradation. On the secondary control level, multi-agent state of charge balancing, reactive power sharing, frequency restoration and voltage restoration are combined, to aggregate energy storage systems for the tertiary control. This includes the novel use of multi-agent sliding mode control for state of charge balancing between AC microgrid energy storage systems. Unlike a linear state of charge balancing strategy, circulating currents are prevented, increasing efficiency and reducing lifetime degradation. An RTDS Technologies real-time digital simulator was used to verify the performance of the proposed control strategy.
\end{abstract}

Index Terms-Battery, dynamic optimal power flow, dynamic programming, energy management, energy storage, microgrid, model predictive control, multi-agent control, state of charge balancing, secondary control, supercapacitor, tertiary control, ultracapacitor.

\section{INTRODUCTION}

$\mathbf{M}$ ICROGRIDS have been proposed for integrating distributed renewable sources and energy storage (ES) systems into power networks [1]. A microgrid is a cluster of generation sources, loads and ES systems that can operate as a dispatchable source in the main grid, or autonomously if islanded.

The traditional hierarchical microgrid control model has three levels [2]. The primary control level is responsible for maintaining the microgrid power balance, to achieve autonomous operation. The most common primary control strategy is decentralised droop control, which provides load sharing

T. Morstyn is with the Department of Engineering Science at the University of Oxford, Oxford OX1 2JD, United Kingdom (email: thomas.morstyn@eng.ox.ac.uk)

A. V. Savkin and B. Hredzak are with the School of Electrical Engineering and Telecommunications at The University of New South Wales (UNSW Australia), Sydney, NSW 2052 Australia (email: a.savkin@unsw.edu.au, b.hredzak@unsw.edu.au).

H. D. Tuan is with the Faculty of Engineering and Information Technology at University of Technology Sydney, Ultimo, NSW 2007, Australia (email: tuan.hoang@uts.edu.au) between sources, without requiring time-critical communication links. A slower centralised secondary control level is introduced to restore frequency and voltage offsets introduced by the primary control, and/or to correct the load sharing ratios between the sources. Finally, on the tertiary control level, the microgrid energy management problem is solved on a slow time-scale. This involves scheduling energy flows between the sources, accounting for renewable generation and load predictions. For microgrids with ES systems, the ES system state of charge (SoC) levels must be managed over long timescales, to ensure they do not run out of energy or become overcharged [3].

The scalability of the energy management problem is of significant concern, since accurate power network models and ES system models make the optimisation non-convex [4], [5]. A common approach is to use an ideal real power transfer model for the microgrid and simplified linear models for the ES system SoC dynamics [6]-[9]. This gives a convex optimisation problem, for which fast and robust solvers are readily available [10].

The ideal real power transfer model used in [6]-[9] is justified for low voltage microgrids with closely located sources and loads, since only a small proportion of overall losses are associated with power distribution [11]. However, ES system charging/discharging losses are significant, and vary nonlinearly with the charge/discharge rate and SoC [12]. Also, for battery ES systems in particular (e.g. lead-acid, lithium ion), depreciation due to lifetime degradation is a significant part of the operating cost [13]. Lifetime degradation has a nonlinear relationship with the battery charge/discharge rate and $\mathrm{SoC}[14]$.

In [15], the microgrid energy management problem, including accurate models for battery ES system efficiency and lifetime degradation, is formulated as a sequential decision making problem. The schedule of energy flows for a given time horizon can be optimised with dynamic programming and backward recursion using Bellman's equation [16].

The main disadvantage of a dynamic programming approach for energy management is the 'curse of dimensionality' [17]. Each additional ES system contributes an extra dimension to the solution space. Dynamic programming involves recursively calculating the cost for all feasible solutions, and thus the computational complexity increases exponentially with the number of ES systems [4].

Another limitation of the traditional hierarchical microgrid 
control model is the centralised structure of the secondary and tertiary control levels. The communications infrastructure required for centralised control limits scalability and flexibility, and may not be cost effective for a microgrid with many small distributed ES systems [18].

This motivates the use of multi-agent control for coordinating microgrid ES systems [19]. Under a multi-agent control strategy, autonomous agents use local information and neighbour-to-neighbour communication over a sparse communication network to achieve cooperative objectives [20]. Multiagent secondary level control strategies have been proposed for microgrid voltage/frequency restoration and load sharing correction [21]-[23].

Multi-agent control has also been proposed for SoC balancing between microgrid ES systems [24]-[29]. Under a SoC balancing strategy, the ES systems share the microgrid load, while moving towards a balanced SoC. Once a balanced SoC has been reached, the combined energy and current capacities of the ES systems are available to maintain power quality and stability in the microgrid. In effect, the distributed ES systems operate as a single larger virtual source of ES.

This paper proposes multi-agent ES system aggregation as a means of scaling energy management to low voltage microgrids with distributed ES systems. Based on this concept, a hierarchical control strategy is developed for an AC microgrid with distributed battery and ultracapacitor ES systems. The novel features of the proposed control strategy are:

1) On the tertiary control level, the energy management problem for microgrids with multiple ES systems is made scalable, by considering each type of ES system in aggregate. This reduces potential degrees of freedom, but has the significant advantages of (i) addressing the 'curse of dimensionality' common to existing dynamic programming approaches, since additional ES systems do not increase the optimisation problem dimension, and (ii) allows nonlinear ES models to be used for the optimisation, accounting for variable efficiency, selfdischarge and lifetime degradation.

2) On the secondary control level, multi-agent $\mathrm{SoC}$ balancing, reactive power sharing, frequency restoration and voltage restoration are combined, to aggregate ES systems into groups that can be scalably controlled by the tertiary level strategy.

3) The multi-agent secondary control level includes the novel use of multi-agent sliding mode control for SoC balancing between AC microgrid ES systems. Unlike a linear SoC balancing strategy, circulating currents are prevented, increasing efficiency and reducing lifetime degradation, since unnecessary charge/discharge cycles are not introduced.

Receding horizon model predictive control (MPC) is used to implement the proposed tertiary level energy management strategy in real-time. The performance of the proposed control strategy was verified with an RTDS Technologies real-time digital simulator, for an AC microgrid with variable photovoltaic $(\mathrm{PV})$ generation, using switching converter models and nonlinear lead-acid battery and ultracapacitor models.

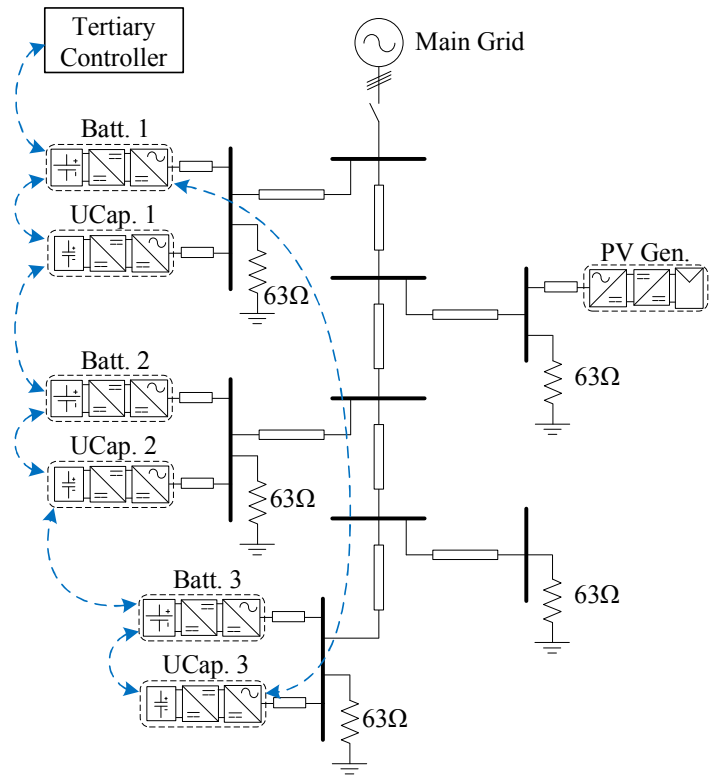

Fig. 1. Low voltage islanded AC microgrid with a PV generation source, three lead-acid battery ES systems and three ultracapacitor ES systems. The ES systems are connected by a sparse communication network.

The rest of this paper is organised as follows. Section II presents the proposed tertiary level energy management strategy for aggregated ES systems. In Section III, the proposed multi-agent secondary level ES system aggregation strategy is presented. Section IV provides real-time digital simulation results, verifying the performance of the proposed control strategy. Section V concludes the paper.

\section{Energy Management for Aggregated Storage SYSTEMS}

This study considers an islanded AC microgrid with two types of distributed ES systems, batteries, labelled type 1, and ultracapacitors, labelled type 2 . Let $\mathcal{V}_{1}=\left\{1, \ldots, N_{1}\right\}$ be the set of battery ES systems, $\mathcal{V}_{2}=\left\{N_{1}+1, \ldots, N_{1}+N_{2}\right\}$ be the set of ultracapacitor ES systems and $\mathcal{V}=\mathcal{V}_{1} \cup \mathcal{V}_{2}$ be the combined set of ES systems.

In particular, the study considers the islanded microgrid shown in Fig. 1, with a PV generation source, three ultracapacitor ES systems and three lead-acid battery ES systems. The microgrid is based on the European residential benchmark low voltage microgrid from [30].

Under the proposed multi-agent secondary level control strategy, presented in Section III, the ES systems of each type operate together as aggregated sources of ES. On the tertiary control level, the microgrid energy management problem is introduced to optimise energy flows between the ES system groups over a time horizon for which renewable generation and load predictions are available. The renewable generation sources are controlled locally for maximum power point tracking, and it is assumed that the microgrid loads are not controllable. 


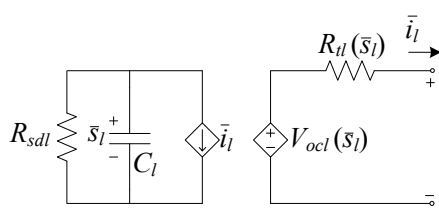

Fig. 2. Equivalent circuit model, applicable to electrochemical batteries (e.g lead-acid, lithium ion) and ultracapacitors.

It is assumed that the ES systems operate cooperatively. This is valid if (i) the distributed ES systems are owned/operated by a single entity, or (ii) individual owners (e.g. homes with local ES systems) are incentivised to cooperate with one another. The proposed energy management strategy has the potential to provide collective value through loss reduction, continuity of supply during islanding and improved ES and renewable generation capacity utilisation. Methods from game theory for distributing this value between microgrid resource owners to incentivise cooperation are discussed in [31].

The proposed control strategy considers groups of ES system in aggregate. In this study, a group of lead-acid batteries and a group of ultracapacitors are considered. Alternative ES system technologies can be accommodated, since the dynamic programming approach does not place restrictions on the optimisation cost function or constraints.

If ES systems with different technologies are added to the microgrid, these can either be organised into additional groups, or groups with a mix of ES technologies can be formed. The number of additional groups is limited, since adding a group increases the problem dimension. Groups made up of ES systems with different parameters, or even different technologies, are allowed, since the dynamic programming problem is based on each group's average SoC, efficiency and lifetime dynamics. This does have limitations, since the ES systems will share the same SoC and per-unit output current (current divided by Ah capacity). Therefore, constraints on these quantities are set by the lowest capacity ES system.

The proposed control strategy is formulated based on an ideal real power transfer model for the microgrid. Therefore, it is most appropriate for low voltage microgrids with closely located ES systems, since the operation of individual ES systems is not differentiated by their network location. The control of low voltage microgrids is an important problem class, since it describes low voltage distribution networks connecting residential/commercial consumers with renewable generation and ES systems, which are playing an increasingly important role in power networks [32]. However, it may not be appropriate for larger microgrids operating at medium voltages, if distribution losses and network power flow constraints are significant.

Fig. 2 shows an equivalent circuit model, which can be applied to electrochemical batteries (e.g lead-acid, lithium ion) [12], and ultracapacitors [33]. Fast output voltage dynamics are neglected, as these do not significantly impact the SoC dynamics.

The average SoC dynamics of each ES system type $l=$

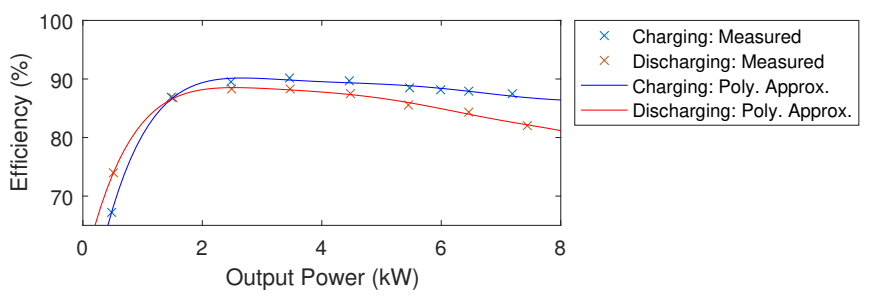

Fig. 3. Charging and discharging efficiency values for $5 \mathrm{~kW}$ DC-DC and DCAC converter stages in series, obtained from an RTDS Technologies real-time digital simulator switching model, and $6^{\text {th }}$ order polynomial approximations.

$\{1,2\}$ can be modelled by the equations,

$$
\begin{aligned}
& \bar{s}_{l}(k+1)=a_{l} \bar{s}_{l}(k)+b_{l} \bar{i}_{l}(k), \\
& a_{l}=e^{-\frac{T_{s}}{C_{l} R_{s d l}}}, b_{l}=-R_{s d l}\left(1-e^{-\frac{T_{s}}{C_{l} R_{s d l}}}\right) .
\end{aligned}
$$

$T_{s}$ is the sampling period of the discrete time model, $\bar{i}_{l}$ is the average output current, $C_{l}$ is the average charge capacity and $R_{s d l}$ models the ES system self-discharge rate.

The average charging/discharging power is given by,

$$
\bar{P}_{l}(k)=V_{o c l}\left(\bar{s}_{l}\right) \bar{i}_{l}-R_{t l}\left(\bar{s}_{l}\right) \bar{i}_{l}^{2} .
$$

$V_{o c l}$ and $R_{t l}$ are SoC dependent open circuit voltage and terminal resistance values.

The ES systems are interfaced with the microgrid through bidirectional DC-DC boost converters and DC-AC voltage source converters (VSC). The power conversion stages introduce additional power dependent losses. The average ES system output power is described by,

$$
\begin{aligned}
& \bar{P}_{o l}(k)= \begin{cases}\left(\frac{1}{1+\epsilon_{0}\left(\bar{P}_{l}\right)}\right) \bar{P}_{l}, & \bar{P}_{l} \geq 0, \\
\left(1+\epsilon_{0}\left(\bar{P}_{l}\right)\right) \bar{P}_{l}, & \bar{P}_{l}<0,\end{cases} \\
& \epsilon_{0}\left(\bar{P}_{l}\right)>0, \forall \bar{P}_{l} \in \mathbf{R} .
\end{aligned}
$$

Fig. 3 shows charging efficiency and discharging efficiency values, $\frac{1}{1+\epsilon_{0}}$, for $5 \mathrm{~kW}$ DC-DC and DC-AC converter stages in series, obtained from an RTDS Technologies real-time digital simulator switching model, as well as $6^{\text {th }}$ order polynomial approximations.

The weighted Ah throughput model from [34] is used to calculate the ES system lifetime degradation,

$$
\begin{aligned}
L_{l}(k+1) & =L_{l}(k)-f_{D l}\left(\bar{s}_{l}(k), \bar{i}_{l}(k)\right), \\
f_{D l}(k) & =\frac{\left(\lambda_{0 l}-\lambda_{1 l} \bar{s}_{l}(k)\right) T_{s}}{N_{l}^{c y c} D o D_{l}^{c y c} C_{l}}\left|\bar{i}_{l}(k)\right| .
\end{aligned}
$$

$L_{l}$ is the normalised ES system lifetime, $N_{l}^{c y c}$ is the number of charge/discharge cycles at the nominal depth of discharge, $D o D_{l}^{c y c}$, before the ES system capacity has degraded to the point of requiring replacement. $\lambda_{0 l}$ and $\lambda_{1 l}$ are fixed coefficients that are used to model the increased lifetime degradation that occurs at lower SoC levels for a given output current. They can be obtained by fitting the model to cyclelife-depth of discharge plots available from battery datasheets. In general, $\lambda_{0 l}>\lambda_{1 l} \geq 0$. Note that the dynamic programming approach does not restrict more detailed models from being used if appropriate data is available. 
Energy management is implemented on the tertiary control level using receding horizon MPC. Let $\mathcal{T}=\left\{k_{0}, \ldots, k_{T}\right\}$ be the optimisation time horizon, where $k_{T}=k_{0}+N_{\text {pred }}-1$. It is assumed that the central processor has access to predictions of the average net microgrid load (i.e. renewable generation minus the total load) for each interval of the time horizon, $P_{n e t}(k), k \in \mathcal{T}$, and estimates of the average SoC for each group of aggregated ES systems, $\bar{s}_{l}\left(k_{0}\right), l \in\{1,2\}$, obtained from the secondary control level.

The optimisation includes the competing objectives of minimising power losses, minimising ES depreciation costs and regulating the aggregated ES system groups near desired $\mathrm{SoC}$ levels. Combining these together motivates the following cost function to minimise,

$$
\begin{aligned}
& \sum_{k=k_{0}}^{k_{T}} \sum_{l=1}^{2}\left[C_{D l} N_{l} f_{D l}+C_{R l} N_{l}\left(\bar{s}_{l}^{*}-\bar{s}_{l}\right)^{2}\right. \\
& \left.+C_{E} N_{l}\left(C_{l} V_{o c l}\left(1-a_{l}\right) \bar{s}_{l}+T_{s} R_{t l} \bar{i}_{l}^{2}+T_{s}\left|\bar{P}_{o l}-\bar{P}_{l}\right|\right)\right] \rightarrow \text { min. }
\end{aligned}
$$

$C_{E}$ is the cost of energy. For ES systems of type $l, C_{D l}$ is the ES system replacement cost and $C_{R l}$ is the cost assigned to squared deviations of the average $\mathrm{SoC}$ from the reference $\bar{s}_{l}^{*}$.

The power balance of the islanded microgrid must be maintained by the ES systems. Therefore,

$$
P_{n e t}(k)=N_{1} \bar{P}_{o 1}(k)+N_{2} \bar{P}_{o 2}(k) .
$$

Combining (2), (3) and (6), the type 2 ES average output current value $\bar{i}_{2}(k)$ can be determined for a given pair of values for $\bar{i}_{1}(k), \bar{s}_{2}(k)$.

Now, the energy management problem can be stated as follows:

Consider the system defined by (1), (2), (3), (6). For a given initial time $k_{0}$, final time $k_{T}$, initial condition $\left(\bar{s}_{1}\left(k_{0}\right), \bar{s}_{2}\left(k_{0}\right)\right)$ satisfying the $\mathrm{SoC}$ constraints $\bar{s}_{l}\left(k_{0}\right) \in\left[\bar{s}_{l}^{\min }, \bar{s}_{l}^{\max }\right], l=$ $\{1,2\}$, and net load predictions $P_{\text {net }}(k), k \in \mathcal{T}$, find the $\bar{i}_{1}(k), k \in \mathcal{T}$, that delivers the minimum value for the cost function (5) while satisfying the microgrid operating constraints.

Functions $V\left(\bar{s}_{1}, \bar{s}_{2}, k\right), k=k_{0}, k_{0}+1, \ldots, k_{T}+1$ are introduced as follows:

$$
\begin{aligned}
& V\left(\bar{s}_{1}, \bar{s}_{2}, k_{T}+1\right)=0, \forall \bar{s}_{1} \in\left[\bar{s}_{1}^{\text {min }}, \bar{s}_{1}^{\max }\right], \bar{s}_{2} \in\left[\bar{s}_{2}^{\min }, \bar{s}_{2}^{\max }\right] . \\
& V\left(\bar{s}_{1}, \bar{s}_{2}, k\right)=\min _{\bar{i}_{1}, \bar{i}_{2}}\left[\sum_{l=1}^{2} C_{D l} N_{l} f_{D l}+C_{R l} N_{l}\left(\bar{s}_{l}^{*}-\bar{s}_{l}\right)^{2}\right. \\
& \quad+C_{E} N_{l}\left(C_{l} V_{o c l}\left(1-a_{l}\right) \bar{s}_{l}+T_{s} R_{t l} \bar{i}_{l}^{2}+T_{s}\left|\bar{P}_{o l}-\bar{P}_{l}\right|\right) \\
& \left.\quad+V\left(a_{1} \bar{s}_{1}+b_{1} \bar{i}_{1}, a_{2} \bar{s}_{2}+b_{2} \bar{i}_{2}, k+1\right)\right], \\
& \text { such that } \bar{s}_{1}(k) \in\left[\bar{s}_{1}^{\text {min }}, \bar{s}_{1}^{\text {max }}\right], \bar{s}_{2}(k) \in\left[\bar{s}_{2}^{\text {min }}, \bar{s}_{2}^{\text {max }}\right], \\
& \quad \bar{i}_{1}(k) \in\left[\bar{i}_{1}^{\text {min }}, \bar{i}_{1}^{\text {max }}\right], P_{n e t}(k)=N_{1} \bar{P}_{o 1}(k)+N_{2} \bar{P}_{o 2}(k), \\
& \quad \forall k=k_{T}, \ldots, k_{0}+1, k_{0} .
\end{aligned}
$$

Constraints $\left[\bar{i}_{1}^{\min }, \bar{i}_{1}^{\max }\right]$ are introduced on the battery ES system output currents, since batteries have low power density relative to ultracapacitors.

The optimisation problem is solved using Bellman's dynamic programming principle [16]. The feasible ranges for the SoC levels are divided into sets of discrete values using suitable step sizes. Then, for $k=k_{T}, k_{T}-1, \ldots, k_{0}$, recursively back in time, step-by-step, the functions in (7) are solved for all allowed pairs of SoC values $\left(\bar{s}_{1}, \bar{s}_{2}\right)$. For each $k$, the minimum value of $V\left(\bar{s}_{1}, \bar{s}_{2}, k\right)$, which is achieved with the optimal control input $\bar{i}_{1}^{\text {opt }}\left(\bar{s}_{1}, \bar{s}_{2}, k\right)$, and corresponding $\bar{i}_{2}^{o p t}\left(\bar{s}_{1}, \bar{s}_{2}, k\right)$ defines the minimum cost-to-go.

The optimal value of the cost function over the full MPC time horizon is given by $V\left(\bar{s}_{1}, \bar{s}_{2}, k_{0}\right)$ and the optimal average output currents of the aggregated ES system groups are defined by the sequence of $\left(\vec{i}_{1}^{\text {opt }}, \vec{i}_{2}^{\text {opt }}\right)$ values for $k=k_{0}, \ldots, k_{T}$. Unlike gradient-based and heuristic methods, the dynamic programming approach recursively evaluates and compares the costs of all feasible solutions [4]. This means that $V\left(\bar{s}_{1}, \bar{s}_{2}, k_{0}\right)$ is the optimal solution of the energy management problem (7). Since the two types of ES systems are considered in aggregate, the optimisation is $2 \mathrm{D}$ and is computationally tractable.

To implement the tertiary control level in real-time, receding horizon MPC is used:

1) The optimal average battery current for the current time interval $\bar{i}_{1}^{o p t}\left(\bar{s}_{1}, \bar{s}_{2}, k_{0}\right)$ is sent as a reference to the lower level secondary control.

2) The MPC time horizon recedes by a step: $k_{0} \leftarrow k_{0}+1$.

3) New predictions for the net microgrid load, $P_{\text {net }}(k), \quad k \in \mathcal{T}$, and estimates for the average battery and ultracapacitor SoC levels, $\bar{s}_{1}\left(k_{0}\right), \bar{s}_{2}\left(k_{0}\right)$, are obtained.

4) The energy management problem is solved for the next time interval.

The references for the ES system groups are calculated based on the assumption of a discrete grid of possible SoC values. Since the battery current reference $\bar{i}_{1}^{\text {opt }}$ is used as the reference sent to the lower level controllers, the average battery $\mathrm{SoC}$ is expected to closely match the desired profile. The lower level control will adjust the ultracapacitor current to maintain the microgrid power balance. If the net load predictions are inaccurate, or the ultracapacitor SoC resolution is insufficient, the average ultracapacitor SoC may deviate from its desired value for a time interval. The main advantage of the receding horizon is that it prevents deviations from accumulating between time intervals.

\section{Multi-Agent Storage System Aggregation}

A multi-agent secondary level control strategy is proposed as a scalable means of aggregating the ES systems for the tertiary control level. The ES system aggregation strategy has the following objectives:

1) Accurate Current Sharing: The average output current for each ES system group matches the optimal reference generated by the tertiary control level. Once a balanced SoC is achieved, the ES systems within each group operate with the same output current.

2) SoC Balancing: The ES systems within each aggregated group move towards a balanced SoC. The ES systems are either all charging or all discharging, so circulating currents are prevented, increasing efficiency and reducing lifetime degradation. 


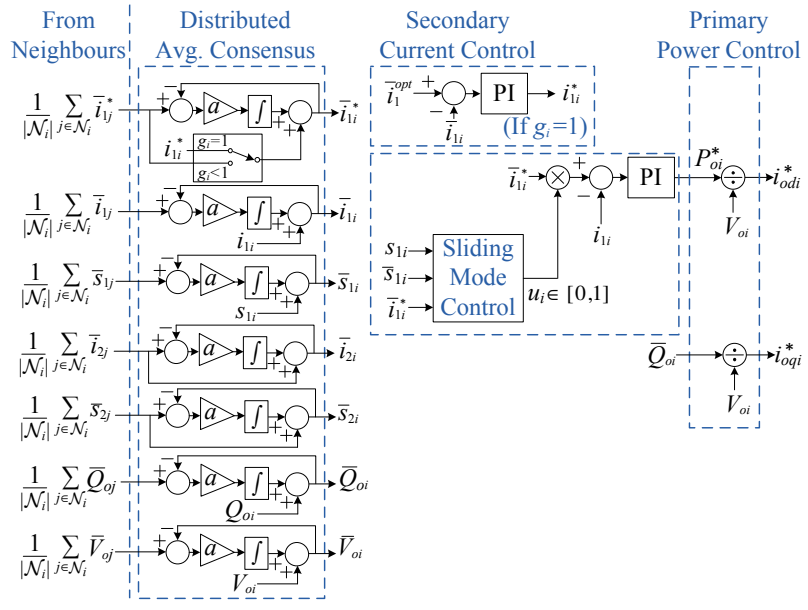

Fig. 4. Primary and secondary level control for one of the battery ES systems. $g_{i}=1$ if the battery is connected to the tertiary level controller, and $g_{i}=0$ otherwise.

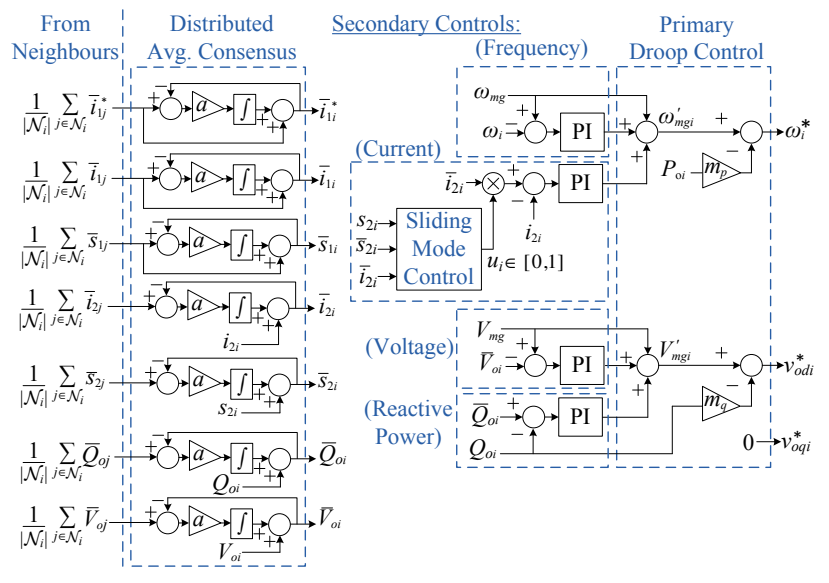

Fig. 5. Primary and secondary level control for one of the ultracapacitor ES systems.

3) Accurate Reactive Power Sharing: The ES systems equally share the microgrid reactive load.

4) Power Quality Regulation: The microgrid frequency and the average of the ES system voltage magnitudes are restored to nominal microgrid reference values.

Below the multi-agent secondary control level, decentralised droop control on the primary control level coordinates load sharing between the ES systems on a fast time-scale, to maintain the microgrid power balance and transient stability. Fig. 4 shows the control strategy for one of the battery ES systems and Fig. 5 shows the control strategy for one of the ultracapacitor ES systems.

\section{A. Decentralised Primary Control}

Qualitatively different operation is desired from the batteries and ultracapacitors. Ultracapacitors have high power density and high cycle life, and thus are suited for supplying the rapid load fluctuations necessary to maintain the microgrid power balance between the sampling intervals of the tertiary level energy management strategy. The batteries have high energy density, but low power density and low cycle life, and should not be used to supply these fluctuations.

The ultracapacitor ES systems operate under the standard $\omega-P, V-Q$ droop control [35]. For ultracapacitor ES system $i \in \mathcal{V}_{2}$,

$$
\begin{aligned}
\omega_{i}^{*} & =\omega_{m g i}^{\prime}-m_{p} P_{o i}, \\
v_{o d i}^{*} & =V_{m g i}^{\prime}-m_{q} Q_{o i}, v_{o q i}^{*}=0 .
\end{aligned}
$$

$\omega_{i}^{*}$ is the VSC frequency reference, $v_{o d i}^{*}$ and $v_{o q i}^{*}$ are the output voltage references in a synchronous d-q reference frame, $P_{o i}$ is the real output power, $Q_{o i}$ is the reactive output power and $m_{p}$ and $m_{q}$ are the droop coefficients. $\omega_{m g}$ and $V_{m g}$ are the nominal microgrid frequency and voltage magnitude. $\omega_{m g i}^{\prime}$ and $V_{m g i}^{\prime}$ are modified versions, set by the multi-agent secondary level control strategy. The frequency and voltage references are sent to the lower level cascaded VSC voltage and current controllers from [35].

The battery ES systems operate as controlled power sources. For battery ES system $i \in \mathcal{V}_{1}$,

$$
i_{o d i}^{*}=\frac{1}{V_{o i}} P_{o i}^{*}, \quad i_{o q i}^{*}=\frac{1}{V_{o i}} \bar{Q}_{o i} .
$$

$i_{o d i}^{*}$ and $i_{o q i}^{*}$ are the $\mathrm{d}-\mathrm{q}$ reference frame output current references, $P_{o i}^{*}$ is the real output power reference generated by the secondary current control strategy, $\bar{Q}_{o i}$ is the local estimate of the average ES system reactive output power and $V_{o i}$ is the output voltage magnitude. The current references are sent to the lower level VSC current control strategy from [35].

\section{B. Multi-Agent Secondary Control for Voltage/Frequency Restoration, Current Sharing and Reactive Power Sharing}

The proposed multi-agent secondary level control strategy for ES system aggregation combines multi-agent sliding mode control for SoC balancing (first presented in [36] for multimodule DC battery ES system SoC balancing) with the multiagent secondary level control strategy for voltage restoration, frequency restoration and reactive power sharing from [23].

The ES systems are connected by a sparse communication network, represented by a connected undirected graph $\mathcal{G}(\mathcal{V}, \mathcal{E})$. $\mathcal{E}$ is the set of graph edges, where the unordered pair $\{i, j\} \in \mathcal{E}$ if there is a communication link between ES system $i$ and $j$. Let $\mathcal{N}_{i}$ be the neighbour set of ES systems $i$, where $j \in \mathcal{N}_{i}$ if $\{i, j\} \in \mathcal{E}$. The requirements on the communication network are that 1) it is connected, meaning that it provides a path between all of the ES systems and 2) it is undirected, meaning that each communication link allows information to flow in both directions.

The ES systems implement the distributed dynamic average consensus protocol from [37], using local information and information from their neighbours to update local estimates of the average currents and SoC levels for each type of ES system $\bar{i}_{1 i}, \bar{i}_{2 i}, \bar{s}_{1 i}, \bar{s}_{2 i}$, the average ES system output voltage $\bar{V}_{o i}$ and the average reactive output power $\bar{Q}_{o i}$. The protocol is modified, so that for ES systems without access to a local 
value of a particular quantity, the average of its neighbours' estimates is passed through instead (e.g. battery SoC $s_{1 i}$ only exists for $i \in \mathcal{V}_{1}$, and not for the ultracapacitor ES systems, $i \in \mathcal{V}_{2}$ ).

Example: For a particular quantity $x_{l i}$, available to ES systems of type $l$, the distributed dynamic average consensus protocol is given by,

$$
\begin{aligned}
\bar{x}_{l i}(t) & =z_{i}(t)+\frac{a}{\left|\mathcal{N}_{i}\right|} \int_{0}^{t} \sum_{j \in \mathcal{N}_{i}}\left(\bar{x}_{l j}(\tau)-\bar{x}_{l i}(\tau)\right) d \tau, \\
z_{i}(t) & = \begin{cases}x_{l i}(t), & i \in \mathcal{V}_{l}, \\
\frac{1}{\left|\mathcal{N}_{i}\right|} \sum_{j \in \mathcal{N}_{i}} \bar{x}_{l j}(t), & \text { otherwise. }\end{cases}
\end{aligned}
$$

$a$ is the average consensus gain and $\left|\mathcal{N}_{i}\right|$ is the number of neighbours of ES system $i$. For step inputs, $x_{l j}, j \in$ $\mathcal{V}_{l}$ and a connected bidirectional communication network, $\lim _{t \rightarrow \infty} \bar{x}_{l i}=\frac{1}{\left|\mathcal{V}_{l}\right|} \sum_{j \in \mathcal{V}_{l}} x_{l j}, \forall i \in \mathcal{V}$.

It is assumed that at least one battery ES system is connected to the tertiary level controller (denoted $g_{i}=1$ ). For the other ES systems, $g_{i}=0$. During each sampling interval of the energy management strategy, the tertiary level controller receives estimates of the average SoC levels for each ES system type, $\bar{s}_{1 i}, \bar{s}_{2 i}$, from its connected battery ES system, and provides it with the optimal average battery current reference for the current sampling interval, $\bar{i}_{1}^{o p t}$.

To ensure that the average battery output current matches $\bar{i}_{1}^{o p t}$, the battery ES systems that are connected to the tertiary controller $\left(g_{i}=1\right)$ generate $i_{1 i}^{*}$ values, using

$$
\begin{aligned}
& i_{1 i}^{*}(t)=k_{1 p}^{\bar{i}^{o p t}} e_{1 i}^{\bar{i}^{o p t}}(t)+k_{1 i}^{\bar{i}^{o p t}} \int_{0}^{t} e_{1 i}^{\bar{i}^{o p t}}(\tau) d \tau, \\
& e_{1 i}^{\bar{i}^{o p t}}(t)=\bar{i}_{1}^{o p t}(t)-\bar{i}_{1 i}(t) .
\end{aligned}
$$

$k_{1 p}^{\bar{i}^{o p t}}$ and $k_{1 i}^{\bar{i}^{o p t}}$ are proportional and integral control gains. Each ES system maintains a local estimate for the average of these values, $\bar{i}_{1 i}^{*}$, using the distributed dynamic average consensus protocol.

To achieve SoC balancing between the ES systems of each type, the local sliding mode controllers generate control signals $u_{i} \in[0,1]$, which set the level of current sharing participation. Full participation is given by $u_{i}=1$. The mechanism for selecting $u_{i}$ is described in Section III-C.

For battery ES system $i \in \mathcal{V}_{1}$, the secondary level control strategy for accurate current sharing is given by,

$$
\begin{aligned}
& P_{o i}^{*}(t)=k_{1 p}^{\bar{i}} e_{1 i}^{\bar{i}_{1}}(t)+\int_{0}^{t} k_{1 i}^{\bar{i}} e_{1 i}^{\bar{i}}(\tau) d \tau, \\
& e_{1 i}^{\bar{i}}(t)=u_{i}(t) \bar{i}_{1 i}^{*}(t)-i_{1 i}(t) .
\end{aligned}
$$

For ultracapacitor ES system $i \in \mathcal{V}_{2}$, the secondary level control strategy for accurate current sharing and frequency restoration is given by,

$$
\begin{gathered}
\omega_{m g i}^{\prime}(t)=\omega_{m g}+k_{p}^{\omega} e_{i}^{\omega}(t)+k_{2 p}^{\bar{i}} e_{2 i}^{\bar{i}}(t) \\
\quad+\int_{0}^{t} k_{i}^{\omega} e_{i}^{\omega}(\tau)+k_{2 i}^{\bar{i}} e_{2 i}^{\bar{i}}(\tau) d \tau, \\
e_{i}^{\omega}(t)=\omega_{m g}-\omega_{i}(t), e_{2 i}^{\bar{i}}(t)=u_{i}(t) \bar{i}_{2 i}(t)-i_{2 i}(t) .
\end{gathered}
$$

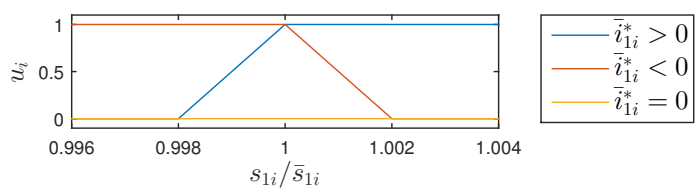

Fig. 6. Battery ES system sliding mode control function $u_{i}\left(s_{1 i}, \bar{s}_{1 i}, \bar{i}_{1 i}^{*}\right)$.

The ultracapacitor ES system secondary level control strategy for average voltage restoration and accurate reactive power sharing is given by,

$$
\begin{gathered}
V_{m g i}^{\prime}(t)=V_{m g}+k_{p}^{\bar{V}} e_{i}^{\bar{V}}(t)+k_{p}^{\bar{Q}} e_{i}^{\bar{Q}}(t) \\
\quad+\int_{0}^{t} k_{i}^{\bar{V}} e_{i}^{\bar{V}}(\tau)+k_{i}^{\bar{Q}} e_{i}^{\bar{Q}}(\tau) d \tau, \\
e_{i}^{\bar{V}}(t)=V_{m g}-\bar{V}_{o i}(t), e_{i}^{\bar{Q}}(t)=\bar{Q}_{o i}(t)-Q_{o i}(t) .
\end{gathered}
$$

\section{Multi-Agent Sliding Mode Control for SoC Balancing}

Multi-agent sliding mode control is introduced to provide SoC balancing between the ES systems. Unlike a linear SoC balancing strategy, circulating currents are prevented, increasing efficiency and reducing lifetime degradation.

For battery ES system $i \in \mathcal{V}_{1}$, the local sliding mode controller generates a control signal $u_{i}$ that determines its current sharing participation level to achieve SoC balancing, based on the local SoC, $s_{1 i}$, its estimate of the average battery SoC, $\bar{s}_{1 i}$, and its estimate of the battery output current reference, $\bar{i}_{1 i}^{*}$.

Note that the ultracapacitor ES systems $i \in \mathcal{V}_{2}$ operate with the same SoC balancing strategy, but with $\left(s_{2 i}, \bar{s}_{2 i}, \bar{i}_{2 i}\right)$ replacing $\left(s_{1 i}, \bar{s}_{1 i}, \bar{i}_{1 i}^{*}\right)$.

First, the following (idealised) sliding mode control function is proposed,

$$
u_{i}(t)= \begin{cases}1, & s_{1 i}(t) \geq \bar{s}_{1 i} \text { and } \bar{i}_{1 i}^{*}(t)>0, \\ 1, & s_{1 i}(t) \leq \bar{s}_{1 i} \text { and } \bar{i}_{1 i}^{*}(t)<0, \\ 0, & \text { otherwise. }\end{cases}
$$

The battery ES system fully participates $\left(u_{i}=1\right)$ if, (a) the battery ES systems are discharging $\left(\bar{i}_{1 i}^{*}>0\right)$ and the local $\mathrm{SoC}$ is greater than, or equal to, the average SoC level, or (b) the ES systems are charging $\left(\bar{i}_{1 i}^{*}<0\right)$ and the local SoC is less than, or equal to, the average SoC level.

A direct implementation of (15) would result in chattering and rapid power fluctuations as the ES systems approached the average SoC level. To prevent this, linear boundary layers are introduced between $s_{1 i}<\bar{s}_{1 i}$ and $s_{1 i}>\bar{s}_{1 i}$. In the boundary layers, the sliding mode control signal is given by a linear interpolation between the values it would otherwise take. Fig. 6 shows the modified implementation of the sliding mode control function.

The tertiary control level generates average battery current references such that the constraints on the average SoC of the ES systems, $\bar{s}_{l}(k) \in\left[\bar{s}_{l}^{\min }, \bar{s}_{l}^{\max }\right], l \in\{1,2\}$, are respected over the time horizon $k \in \mathcal{T}$. The multi-agent sliding mode control strategy prevents the individual ES systems of each type, $l \in\{1,2\}$, from being overcharged/undercharged, since 
TABLE I

CAse Study Parameters

\begin{tabular}{llllll}
\hline$N_{\text {pred }}$ & 12 & $T_{s}$ & $5 \mathrm{~min}$ & $N_{1}$ & 3 \\
$C_{1}$ & $25 \mathrm{Ah}$ & $R_{\text {sd1 }}$ & $775 \Omega$ & $E_{1}$ & $10 \mathrm{kWh}$ \\
$V_{\text {ocl }}^{\text {ful }}$ & $400 \mathrm{~V}$ & $\bar{s}_{1}^{\text {min }}$ & $40 \%$ & $\bar{s}_{1}^{\text {max }}$ & $100 \%$ \\
$\bar{i}_{1}^{\text {min }}$ & $-5.5 \mathrm{~A}$ & $\bar{i}_{1}^{\text {max }}$ & $5.5 \mathrm{~A}$ & $N_{1}^{\text {cyc }}$ & 390 \\
$D_{o} D_{1}^{\text {cyc }}$ & $50 \%$ & $\lambda_{11}$ & 1.6 & $\lambda_{01}$ & 2.1 \\
$C_{D 1}$ & $\$ 2000$ & $C_{R 1}$ & $\$ 0 /(\Delta \mathrm{Ah})^{2}$ & $C_{E}$ & $\$ 0.20 / \mathrm{kWh}$ \\
$N_{2}$ & 3 & $C_{2}$ & $15 \mathrm{Ah}$ & $R_{s d 2}$ & $24 \Omega$ \\
$E_{2}$ & $3 \mathrm{kWh}$ & $V_{o c 2}^{\text {full }}$ & $400 \mathrm{~V}$ & $\bar{s}_{2}^{\text {min }}$ & $50 \%$ \\
$\bar{s}_{2}^{\text {max }}$ & $100 \%$ & $\bar{s}_{2}^{*}$ & $80 \%$ & $C_{D 2}$ & $\$ 0$ \\
$C_{R 2}$ & $\$ 0.05 /(\Delta \mathrm{Ah})^{2}$ & $\omega_{m g}$ & $2 \pi \cdot 50 \mathrm{rad} / \mathrm{s}$ & $V_{m g}$ & $415 \mathrm{~V}$ \\
$m_{p}$ & $4 \cdot 10^{-5}$ & $m_{q}$ & $6 \cdot 10^{-4}$ & $a$ & 200 \\
$k_{1}^{\bar{i}_{p} \text { opt }}$ & 0.01 & $k_{1 i}^{\bar{i}^{\text {opt }}}$ & 1 & $k_{1 p}^{\bar{i}}$ & 100 \\
$k_{1}^{\bar{i}}$ & 1000 & $k_{p}^{\omega}$ & 5 & $k_{i}^{\omega}$ & 30 \\
$k_{2 p}^{i}$ & 0.005 & $k_{2 i}^{i}$ & 0.02 & $k_{p}^{\bar{V}}$ & 0.5 \\
$k_{i}^{\bar{i}}$ & 5 & $k_{p}^{\bar{Q}}$ & 0 & $k_{i}^{\bar{Q}}$ & 0.05 \\
\hline
\end{tabular}

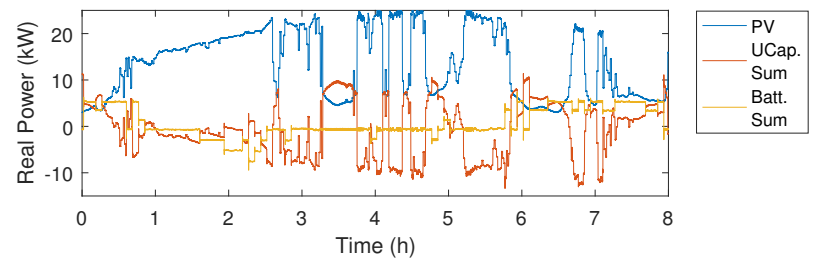

Fig. 7. Microgrid PV generation and the sums of the battery and ultracapacitor ES system real output powers.

they are only charged if their $\mathrm{SoC}$ is less than, or equal to, their estimate of the average $\mathrm{SoC}\left(s_{l i} \leq \bar{s}_{l i}\right)$, and discharged if their SoC is greather than, or equal to, their estimate of the average $\mathrm{SoC}\left(s_{l i} \geq \bar{s}_{l i}\right)$.

\section{RESULTS}

To verify the performance of the proposed control strategy, it was implemented for the islanded AC microgrid, shown in Fig. 1, using an RTDS Technologies real-time digital simulator. The microgrid is based on the European residential benchmark low voltage microgrid from [30]. Switching converter models and nonlinear lead-acid battery and ultracapacitor models capturing fast and slow time-scale voltage and $\mathrm{SoC}$ dynamics were used, allowing the proper interaction between the different microgrid control levels to be verified. The microgrid, as well as the primary and secondary level controllers are implemented on the real-time digital simulator. The tertiary level controller is implemented in MATLAB running on an Intel Core i76500U CPU with 8GB of RAM, and the references generated are supplied to the real-time digital simulator using TCP/IP communication.

The microgrid has $22 \mathrm{~kW}$ nominal PV generation, five $63 \Omega$ loads $(5 \times 2.73 \mathrm{~kW}$ at $415 \mathrm{~V})$, three $10 \mathrm{kWh}$ lead-acid battery ES systems and three $3 \mathrm{kWh}$ ultracapacitor ES systems.

The lead-acid batteries have increased lifetime degradation and reduced efficiency at low SoC levels and high charging/discharging currents. The batteries are assigned SoC limits from $40 \%$ to $100 \%$ and average current limits of $\pm 5.5 \mathrm{~A}$ $\left( \pm 2.2 \mathrm{~kW}\right.$ at $\left.V_{o c 1}^{\text {full }}=400 \mathrm{~V}\right)$.

The ultracapacitor open circuit voltage varies linearly with the $\mathrm{SoC}$ (i.e. $V_{o c 2}\left(\bar{s}_{2}\right)=\bar{s}_{2} V_{o c 2}^{f u l l}$ ). To prevent the voltage from

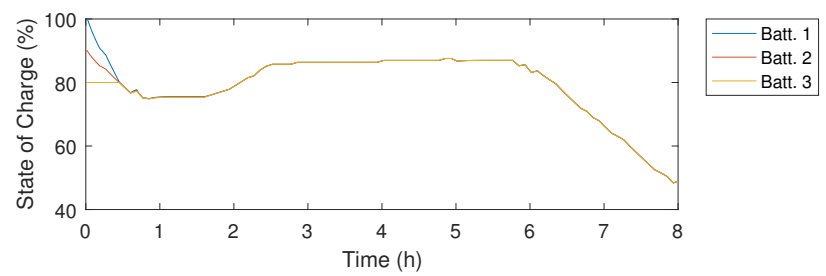

Fig. 8. Battery SoC levels.

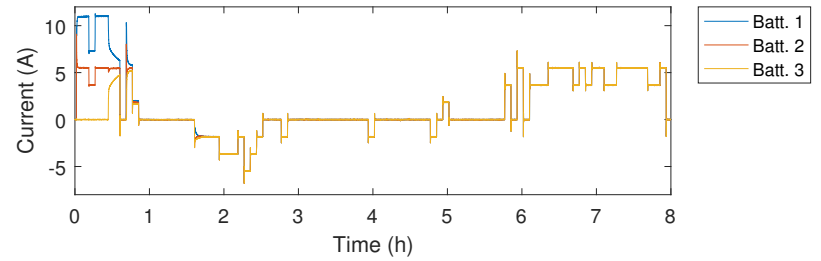

Fig. 9. Battery output currents.

falling too low for proper converter operation, the ultracapacitors are assigned SoC limits from $50 \%$ to $100 \%$. Note that the energy stored in an ultracapacitor is given by, $\frac{1}{2} C_{2} V_{o c 2}^{f u l l} \bar{s}_{2}^{2}$, so that between $100 \%$ and $50 \%$ SoC, $75 \%$ of the stored energy is used.

The cycle life of the lead-acid batteries is 390 cycles, at a nominal depth of discharge of 50\% [34]. The effect of variable depth of discharge on lifetime degradation is modelled by $\lambda_{01}$ and $\lambda_{11}$. Ultracapacitor cycle life is on the order of 1,000,000 cycles [38]. Therefore, only battery depreciation is considered in this study.

Since the ultracapacitors are responsible for maintaining the microgrid power balance, they should maintain spare capacity to deal with unknown future demand fluctuations. To achieve this, an ultracapacitor reference $\mathrm{SoC}$ level of $\bar{s}_{2}^{*}=80 \%$ is used, along with a regulation cost for squared deviations from this value. The battery ES systems are not assigned a reference SoC level or regulation cost.

The PV generation source maximum power point was calculated using one minute resolution temperature and irradiance data from the NREL Baseline Measurement System in Colorado. The data is for $8 \mathrm{am}$ to $4 \mathrm{pm}$ on July $2^{\text {nd }} 2015$.

To allow the battery ES systems to adjust for variations in PV generation, a 5 minute MPC sampling interval is selected. A 1 hour prediction horizon (12 intervals) is used, based on the expected availability of PV predictions from sky imaging sensors [39].

Fig. 7 shows the total real output power for the PV generation source, the battery ES systems and the ultracapacitor ES systems. As shown, the battery ES systems supply the bulk energy requirement of the microgrid, while the ultracapacitor ES systems supply the fluctuations required to maintain the microgrid power balance.

As shown in Fig. 8, the battery ES systems begin with SoC levels between $80 \%$ and $100 \%$. Fig. 9 shows the battery output currents. Fig. 10 shows the ultracapacitor ES system SoC levels, which begin between $70 \%$ and $90 \%$, and Fig. 11 shows the ultracapacitor output currents. 


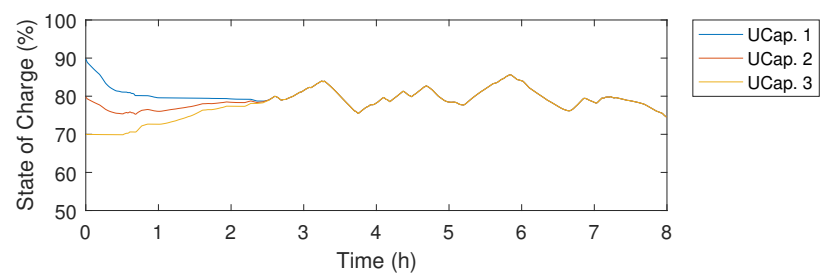

Fig. 10. Ultracapacitor SoC levels.

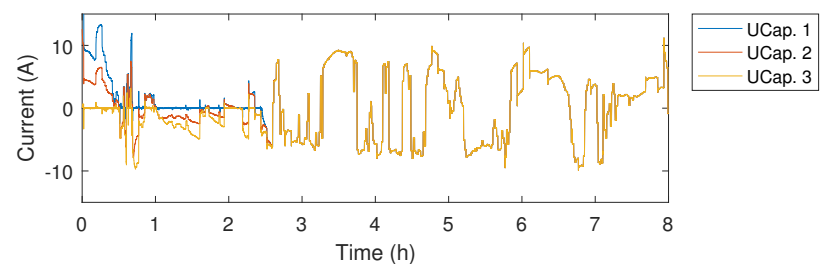

Fig. 11. Ultracapacitor output currents.

The tertiary level energy management strategy sets the reference for the average battery ES system current every 5 minutes. Initially, the battery ES systems and the ultracapacitor ES systems are discharging. Under the secondary level multiagent sliding mode control strategy, the ES systems with high $\mathrm{SoC}$ operate at a high discharging current, while the ES systems with low SoC operate at zero output current. This provides fast SoC balancing, without introducing circulating currents, which would reduce efficiency and increase lifetime degradation.

Once the battery ES systems and ultracapacitor ES systems have reached balanced SoC levels, they operate as aggregated groups of ES. The average of the battery output currents remains within the limits of $\pm 5.5 \mathrm{~A}$. Also, the battery ES systems and ultracapacitor ES systems remain within their respective SoC limits of $40 \%$ to $100 \%$ and $50 \%$ to $100 \%$.

Fig. 12 shows that the microgrid frequency is regulated near the $\omega_{m g}=50 \mathrm{~Hz}$ nominal level, and Fig. 13 shows that the average ES system output voltage is regulated near $V_{m g}=$ $415 \mathrm{~V}$. As shown in Fig. 14, the PV generation source operates at zero reactive power, while the ES systems equally share the reactive load.

Table II compares the computational complexity of the tertiary level energy management strategy, for different problem sizes. The optimisation problem is solved in MATLAB running on an Intel Core i7-6500U CPU with 8GB of RAM. The optimisation was run for the first case study sampling interval using different temporal resolutions, $\Delta T$, (1 $\mathrm{min}, 5 \mathrm{~min}, 20 \mathrm{~min})$ for the 1 hour prediction horizon, and different values for the battery SoC resolution, $\Delta \bar{s}_{1},(0.6 \%, 1.2 \%, 2.4 \%)$. The ultracapacitor $\mathrm{SoC}$ resolution is set at $\Delta \bar{s}_{2}=0.1 \%$. Row 5, indicated in bold, shows the values selected for the tertiary level strategy during the real-time simulation case study.

The computational cost of the dynamic programming approach is mainly from the value function calculations required during the back-stepping procedure, described in (7). For each time interval, $V$ must be calculated for each feasible pair of battery and ultracapacitor $\mathrm{SoC}$ values at that time

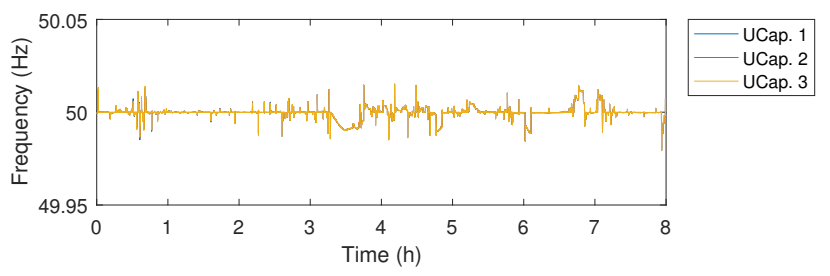

Fig. 12. Ultracapacitor ES system frequencies.

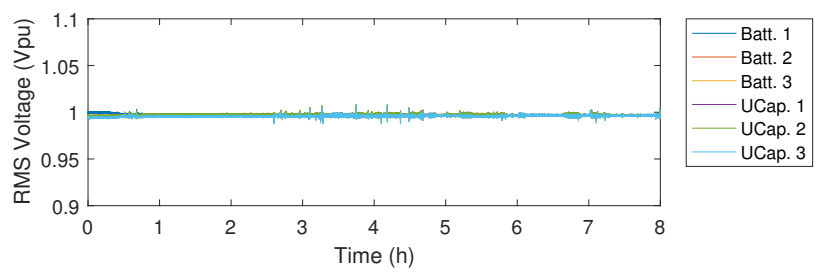

Fig. 13. ES system RMS output voltages.

TABLE II

COMPUTATIONAL COMPLEXITY COMPARISON

\begin{tabular}{rrrrrrrr}
\hline $\begin{array}{r}\Delta T \\
(\mathrm{~min})\end{array}$ & $N_{\text {pred }}$ & $\begin{array}{r}\Delta \bar{s}_{1} \\
(\%)\end{array}$ & $N_{\bar{s}_{1}}$ & $\begin{array}{r}\Delta \bar{s}_{2} \\
(\%)\end{array}$ & $N_{\bar{s}_{2}}$ & $\begin{array}{r}\text { Back-step } \\
V \text { Calcs. }\end{array}$ & $\begin{array}{r}\text { Comp. } \\
\text { Time (s) }\end{array}$ \\
\hline 20 & 3 & 2.4 & 25 & 0.1 & 500 & $0.94 \times 10^{6}$ & 6 \\
20 & 3 & 1.2 & 50 & 0.1 & 500 & $3.75 \times 10^{6}$ & 25 \\
20 & 3 & 0.6 & 100 & 0.1 & 500 & $15.0 \times 10^{6}$ & 94 \\
5 & 12 & 2.4 & 25 & 0.1 & 500 & $3.75 \times 10^{6}$ & 28 \\
$\mathbf{5}$ & $\mathbf{1 2}$ & $\mathbf{1 . 2}$ & $\mathbf{5 0}$ & $\mathbf{0 . 1}$ & $\mathbf{5 0 0}$ & $\mathbf{1 5 . 0 \times 1 0 ^ { 6 }}$ & $\mathbf{1 0 7}$ \\
5 & 12 & 0.6 & 100 & 0.1 & 500 & $60.0 \times 10^{6}$ & 421 \\
1 & 60 & 2.4 & 25 & 0.1 & 500 & $18.75 \times 10^{6}$ & 142 \\
1 & 60 & 1.2 & 50 & 0.1 & 500 & $75.0 \times 10^{6}$ & 561 \\
1 & 60 & 0.6 & 100 & 0.1 & 500 & $300.0 \times 10^{6}$ & 2251 \\
\hline
\end{tabular}

interval, considering each feasible battery $\mathrm{SoC}$ value for the preceding interval (note that these three values determine the ultracapacitor $\mathrm{SoC}$ at the preceding interval). This requires $N_{\text {pred }} \cdot N_{\bar{s}_{1}}^{2} \cdot N_{\bar{s}_{2}}$ calculations. For the selected time horizon and ES system SoC resolutions, $15 \times 10^{6}$ value function calculations are required. Note that increasing the number of battery and/or ultracapacitor ES systems does not increase the computation time, since each type is considered in aggregate by the proposed tertiary level energy management strategy.

Without ES system aggregation, dynamic programming becomes infeasible as the number of ES systems increases. For example, without the proposed aggregation strategy, for the time horizon, equivalent SoC resolutions and ES systems (3 batteries and 3 ultracapacitors) used in the case study, $N_{\text {pred }}\left(\frac{N_{\bar{s}_{1}}}{3}\right)^{6}\left(\frac{N_{\bar{s}_{2}}}{3}\right)^{5}=3.3 \times 10^{19}$ value function calculations would be required.

\section{CONClusion}

A scalable strategy for microgrid energy management has been presented, based on multi-agent ES system aggregation. Additional ES systems added to the microgrid join groups of aggregated ES systems, and thus do not increase the optimisation problem dimension. This addresses the 'curse of dimensionality' which is otherwise the main disadvantage of dynamic programming for microgrid energy management. 


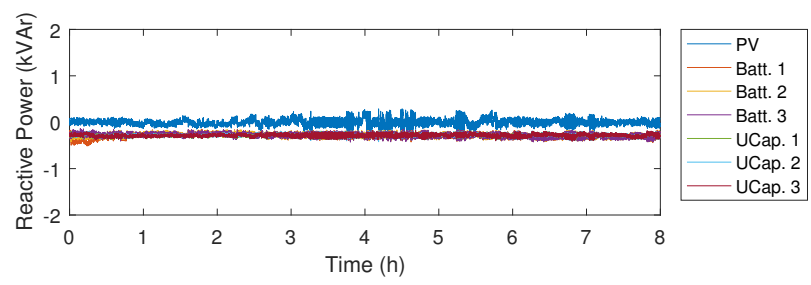

Fig. 14. PV system, battery ES system and ultracapacitor ES system reactive output powers.

Multi-agent secondary level control combining SoC balancing, reactive power sharing, frequency restoration and voltage restoration, is used to provide flexible and scalable ES system aggregation. The secondary control level requires only neighbour-to-neighbour communication over a sparse network. An RTDS Technologies real-time digital simulator was used to verify the proper interaction of the primary, secondary and tertiary control levels.

Future work will consider how to develop the proposed control strategy for large microgrids operating at higher voltages, for which losses and network power flow constraints may be significant. In this case, the ideal real power transfer model used to formulate the tertiary control strategy may no longer be suitable. A promising approach is to combine the proposed dynamic programming strategy for aggregated ES system groups and the method from [4], where for each set of energy flows considered between the ES systems, a power flow solver is used to include losses in the optimisation cost function, and to exclude solutions violating power flow limits. This is possible since a dynamic programming approach does not place restrictions on the optimisation cost function and constraints.

\section{REFERENCES}

[1] R. H. Lasseter, "Smart Distribution: Coupled Microgrids," Proceedings of the IEEE, vol. 99, no. 6, pp. 1074-1082, June 2011.

[2] J. M. Guerrero, J. C. Vásquez, J. Matas, L. G. de Vicuna, and M. Castilla, "Hierarchical Control of Droop-Controlled AC and DC Microgrids-A General Approach Toward Standardization," IEEE Trans. Ind. Electron., vol. 58, no. 1, pp. 158-172, Jan. 2011.

[3] T. Morstyn, B. Hredzak, and V. G. Agelidis, "Control Strategies for Microgrids with Distributed Energy Storage Systems: An Overview," IEEE Trans. Smart Grid, Dec. 2016.

[4] Y. Levron, J. M. Guerrero, and Y. Beck, "Optimal Power Flow in Microgrids With Energy Storage," IEEE Trans. Power Syst., vol. 28, no. 3, pp. 3226-3234, Aug. 2013.

[5] D. E. Olivares, C. A. Canizares, and M. Kazerani, "A Centralized Energy Management System for Isolated Microgrids," IEEE Trans. Smart Grid, vol. 5, no. 4, pp. 1864-1875, July 2014.

[6] E. Mayhorn, K. Kalsi, M. Elizondo, N. Samaan, and K. Butler-Purry, "Optimal control of distributed energy resources using model predictive control," in 2012 IEEE Power and Energy Society General Meeting, July 2012, pp. 1-8.

[7] Q. Jiang, M. Xue, and G. Geng, "Energy management of microgrid in grid-connected and stand-alone modes," IEEE Trans. Power Syst., vol. 28, no. 3, pp. 3380-3389, 2013.

[8] R. Palma-Behnke, C. Benavides, F. Lanas, B. Severino, L. Reyes, J. Llanos, and D. Saez, "A Microgrid Energy Management System Based on the Rolling Horizon Strategy," IEEE Trans. Smart Grid, vol. 4, no. 2, pp. 996-1006, June 2013.

[9] E. Perez, H. Beltran, N. Aparicio, and P. Rodriguez, "Predictive Power Control for PV Plants With Energy Storage," IEEE Trans. Sustain. Energy, vol. 4, no. 2, pp. 482-490, Apr. 2013.
[10] S. Boyd and L. Vandenberghe, Convex Optimization. Cambridge: Cambridge University Press, 2004.

[11] M. Farasat and A. Arabali, "Voltage and power control for minimising converter and distribution losses in autonomous microgrids," IET Generation, Transmission \& Distribution, vol. 9, no. 13, pp. 1614-1620, 2015.

[12] M. Chen and G. A. Rincon-Mora, "Accurate Electrical Battery Model Capable of Predicting Runtime and IV Performance," IEEE Trans. Energy Convers., vol. 21, no. 2, pp. 504-511, June 2006.

[13] J. P. Barton and D. G. Infield, "Energy Storage and Its Use With Intermittent Renewable Energy," IEEE Trans. Energy Convers., vol. 19, no. 2, pp. 441-448, June 2004.

[14] R. Dufo-López, J. M. Lujano-Rojas, and J. L. Bernal-Agustín, "Comparison of different leadacid battery lifetime prediction models for use in simulation of stand-alone photovoltaic systems," Applied Energy, vol. 115, pp. 242-253, Nov. 2013.

[15] T. A. Nguyen and M. L. Crow, "Stochastic Optimization of RenewableBased Microgrid Operation Incorporating Battery Operating Cost," IEEE Trans. Power Syst., vol. 31, no. 3, pp. 2289-2296, May 2016.

[16] R. E. Bellman, "The Theory of Dynamic Programming," Bulletin of the American Mathematical Society, vol. 60, no. 6, pp. 503-515, 1954.

[17] W. B. Powell and S. Meisel, "Tutorial on Stochastic Optimization in Energy - Part II: An Energy Storage Illustration," IEEE Trans. Power Syst., vol. 31, no. 2, pp. 1-8, May 2015.

[18] D. J. Hill, T. Liu, and G. Verbic, "Smart grids as distributed learning control," in 2012 IEEE Power and Energy Society General Meeting, July 2012, pp. 1-8.

[19] S. D. J. McArthur, E. M. Davidson, V. M. Catterson, A. L. Dimeas, N. D. Hatziargyriou, F. Ponci, and T. Funabashi, "Multi-Agent Systems for Power Engineering Applications-Part I: Concepts, Approaches, and Technical Challenges," IEEE Trans. Power Syst., vol. 22, no. 4, pp. 1743-1752, Nov. 2007.

[20] F. L. Lewis, H. Zhang, K. Hengster-Movric, and A. Das, Cooperative Control of Multi-Agent Systems, ser. Communications and Control Engineering. London: Springer London, 2014.

[21] A. Bidram, A. Davoudi, F. L. Lewis, and J. M. Guerrero, "Distributed Cooperative Secondary Control of Microgrids Using Feedback Linearization," IEEE Trans. Power Syst., vol. 28, no. 3, pp. 3462-3470, Aug. 2013.

[22] J. Schiffer, T. Seel, J. Raisch, and T. Sezi, "Voltage Stability and Reactive Power Sharing in Inverter-Based Microgrids with ConsensusBased Distributed Voltage Control," IEEE Trans. Control Syst. Technol., vol. 24, no. 1, pp. 96-109, 2016.

[23] Q. Shafiee, V. Nasirian, J. C. Vásquez, and J. M. Guerrero, "A MultiFunctional Fully Distributed Control Framework for AC Microgrids," IEEE Trans. Smart Grid, Oct. 2016.

[24] T. Morstyn, B. Hredzak, and V. G. Agelidis, "Distributed Cooperative Control of Microgrid Storage," IEEE Trans. Power Syst., vol. 30, no. 5, pp. 2780-2789, Sept. 2015.

[25] T. Morstyn, B. Hredzak, G. D. Demetriades, and V. G. Agelidis, "Unified Distributed Control for DC Microgrid Operating Modes," IEEE Trans. Power Syst., vol. 31, no. 1, pp. 802-812, Jan. 2016.

[26] T. Morstyn, B. Hredzak, and V. G. Agelidis, "Cooperative MultiAgent Control of Heterogeneous Storage Devices Distributed in a DC Microgrid," IEEE Trans. Power Syst., vol. PP, no. 99, pp. 1-13, 2015.

[27] T. Morstyn, B. Hredzak, V. G. Agelidis, and G. Demetriades, "Cooperative control of DC microgrid storage for energy balancing and equal power sharing," in 2014 Australasian Universities Power Engineering Conference (AUPEC), Sept. 2014, pp. 1-6.

[28] T. Morstyn, B. Hredzak, and V. G. Agelidis, "Communication delay robustness for multi-agent state of charge balancing between distributed AC microgrid storage systems," in 2015 IEEE Conference on Control Applications (CCA), Sept. 2015, pp. 181-186.

[29] C. Li, T. Dragičević, M. G. Plaza, F. Andrade, J. C. Vásquez, and J. M. Guerrero, "Multiagent based distributed control for state-of-charge balance of distributed energy storage in DC microgrids," in IECON 2014 - 40th Annual Conference of the IEEE Industrial Electronics Society, Oct. 2014, pp. 2180-2184.

[30] S. Papathanassiou, N. Hatziargyriou, and K. Strunz, "A Benchmark Low Voltage Microgrid Network," in Proceedings of the CIGRE Symposium: Power Systems with Dispersed Generation, no. Jan., 2005, pp. 1-8.

[31] W. Saad, Z. Han, H. V. Poor, and T. Başar, "Game-theoretic methods for the smart grid: An overview of microgrid systems, demand-side management, and smart grid communications," IEEE Signal Processing Magazine, vol. 29, no. 5, pp. 86-105, Sept. 2012. 
[32] C. Restrepo, A. Salazar, H. Schweizer, and A. Ginart, "Residential Battery Storage: Is the Timing Right?" IEEE Electrification Magazine, vol. 3, no. 3, pp. 14-21, Sept. 2015.

[33] R. L. Spyker and R. M. Nelms, "Classical Equivalent Circuit Parameters for a Double-Layer Capacitor," IEEE Trans. Aerosp. Electron. Syst., vol. 36, no. 3, pp. 829-836, Aug. 2000.

[34] B. Zhao, X. Zhang, J. Chen, C. Wang, and L. Guo, "Operation optimization of standalone microgrids considering lifetime characteristics of battery energy storage system," IEEE Trans. Sustain. Energy, vol. 4, no. 4, pp. 934-943, Oct. 2013.

[35] N. Pogaku, M. Prodanovic, and T. C. Green, "Modeling, Analysis and Testing of Autonomous Operation of an Inverter-Based Microgrid," IEEE Trans. Power Electron., vol. 22, no. 2, pp. 613-625, Mar. 2007.

[36] T. Morstyn, A. V. Savkin, B. Hredzak, and V. G. Agelidis, "Distributed Sliding Mode Control for Multi-Module Battery Energy Storage System State of Charge Balancing," in 2016 IEEE Conference on Control Applications (CCA), Sept. 2016, pp. $47-51$.

[37] R. Olfati-saber, D. P. Spanos, and R. M. Murray, "Dynamic Consensus for Mobile Networks," in 2005 IFAC World Congress, July 2005.

[38] A. M. Gee, F. V. P. Robinson, and R. W. Dunn, "Analysis of Battery Lifetime Extension in a Small-Scale Wind-Energy System Using Supercapacitors," IEEE Trans. Energy Convers., vol. 28, no. 1, pp. 24-33, Mar. 2013.

[39] Y. Chu, H. T. C. Pedro, L. Nonnenmacher, R. H. Inman, Z. Liao, and C. F. M. Coimbra, "A Smart image-based cloud detection system for intrahour solar irradiance forecasts," Journal of Atmospheric and Oceanic Technology, vol. 31, no. 9, pp. 1995-2007, Sept. 2014.

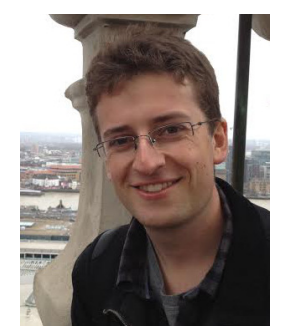

Thomas Morstyn (S'13) received the B.E. (Hon.) degree from the University of Melbourne, Australia, in 2011, and the $\mathrm{PhD}$ degree from the University of New South Wales, Australia, in 2016, both in electrical engineering.

He worked as an electrical engineer in the Rio Tinto Technology and Innovation group between 2012 and 2014. He is currently an Oxford Martin Fellow with the Department of Engineering Science at the University of Oxford. His current research interests include multi-agent control and optimisation for the integration of distributed renewable generation and energy storage systems into power networks.

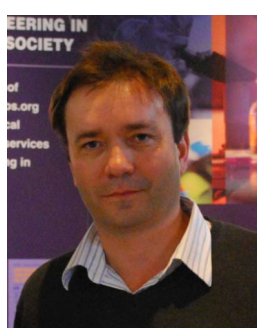

Andrey V. Savkin (M'97-SM'98) received the M.S. degree in mathematics and the Ph.D. degree in applied mathematics from The Leningrad University, Leningrad, Russia, in 1987 and 1991, respectively. Since 2000, he has been a Professor in the School of Electrical Engineering and Telecommunications, The University of New South Wales, Sydney, NSW, Australia. His current research interests include robust control and filtering, robotics, networked control systems, control of power systems and the application of control and signal processing to biomedical engineering and medicine. Dr. Savkin has been an associate editor for several international journals and conferences.

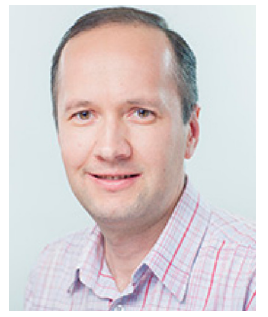

Branislav Hredzak (M'98-SM'13) received the B.Sc./M.Sc. degree from the Technical University of Kosice, Slovak Republic, in 1993, and the Ph.D. degree from Napier University of Edinburgh, U.K., in 1997, all in electrical engineering.

He was a Lecturer and a Senior Researcher in Singapore from 1997 to 2007. He is currently a Senior Lecturer in the School of Electrical Engineering and Telecommunications, The University of New South Wales, Sydney, NSW, Australia. His current research interests include hybrid storage technologies and advanced control systems for power electronics and storage systems.

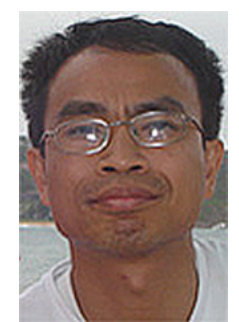

Hoang D. Tuan (M'94) was born in Hanoi, Vietnam. He received the Diploma (Hons.) and Ph.D. degrees in applied mathematics from Odessa State University, Ukraine, in 1987 and 1991, respectively. He spent nine academic years in Japan as an Assistant Professor with the Department of ElectronicMechanical Engineering, Nagoya University, from 1994 to 1999, and then as an Associate Professor with the Department of Electrical and Computer Engineering, Toyota Technological Institute, Nagoya, from 1999 to 2003. He was a Professor with the School of Electrical Engineering and Telecommunications, University of New South Wales, from 2003 to 2011 . He is currently a Professor with the Centre for Health Technologies, University of Technology Sydney. He has been involved in research with the areas of optimization, control, signal processing, wireless communication, and biomedical engineering for more than 20 years. 